\title{
A BUFFERED INTERACTION BETWEEN SEX RATIO, AGE DIFFERENCE AT MARRIAGE, AND POPULATION GROWTH IN HUMANS, AND THEIR SIGNIFICANCE FOR SEX RATIO EVOLUTION
}

\author{
AVIGDOR BEILES \\ Department of Genetics, The Hebrew University of Jerusalem, \\ Jerusalem, Israel
}

Received 1.i.74

\section{Summary}

The presence, in man, of a significant high sex ratio at birth $\left(M_{b}\right)$ is a biological fact, and its evolution is not satisfactorily explained by differential mortality of males.

Such a high sex ratio could have evolved primarily as a buffering interaction between growth rate or increase of human population $\left(R_{b}\right)$ and age difference at marriage $(d)$. The interaction of $R_{b}$ with $d$ will transform a high $M_{b}$ to a lower sex ratio at mating $\left(M_{m}\right)$.

The model developed to account for the interaction also shows that $d$ is able to adjust and to achieve a balanced $M_{m}$ over a relatively broad range. The limits set on $d$ are both biological and cultural; in practically all cultures males father children when they are older than their spouses. A buffered range develops where the balanced $M_{m}$ is maintained in spite of temporal fluctuations or genetic fixations in $M_{b}, R_{b}$, and some additional factors.

A realistic necessary condition for the operation of the buffered system is the combination of $R_{b}>1 \cdot 00, d>0$, and $M_{b}>100$. Buffering of male excesses will be achieved more efficiently than buffering of female excesses, i.e. the range is asymmetrical.

Inside the range, selective pressures will be caused by the longer generation time of the males, rather than by unmated excess of either sex.

According to prevailing fitness models, females should be born in excess, since the longer generation time of males is disadvantageous and should be balanced by the advantage of being the rare sex. That males in fact are born in excess demonstrates a larger significant discrepancy with fitness models than previously assumed.

The buffering of sex ratio by age difference at mating is advantageous mainly in populations which are organized in small groups, as it was in early human history. This pattern also facilitated group selection.

The buffered system herein described evolved in growing populations. In declining populations it causes a cumulative maximal increase of $d$, and severe social problems.

\section{INTRODUCTION}

THE observed sex ratio in man does not exactly fit the expected segregational ratio of $1: 1$. In many populations male births exceed those of females by approximately 106:100, a " high" sex ratio. Information derived from the record of stillbirths widens the discrepancy still further, although recent reports of an opposite trend in spontaneous and induced abortions at the first trimester of pregnancy (Stevenson and Bobrow, 1967; Mikamo, 1969; Keller, 1969; Sasaki et al., 1971; Hahnemann, 1973), raise some doubt as to the overall magnitude and direction of stillbirth effects on the sex ratio. Nevertheless, the weight of current evidence still suggests that the primary sex ratio, i.e. at conception, is probably skewed even more in favour of males 
(Cavalli-Sforza and Bodmer, 1971; Stern, 1973). These facts indicate that the classic 1:1 segregational ratio is subject to some modifying mechanism, as was pointed out, for example, in the review by Cavalli-Sforza and Bodmer (1971). This modification is apparently under genetic control. In other words, one may conclude that the secondary sex ratio (at birth) in man is a product of evolutionary processes.

Several workers have dealt theoretically with the determination of sex ratio. Their analyses have led to the prediction of an equality between the two sexes, or even to a tendency toward an excess of females (see Eshel, 1973, and reviews by Edwards, 1962, and Cavalli-Sforza and Bodmer, 1971). Hamilton (1967) and Eshel (1973) have concluded that even a high sex ratio due to sex-linked modifiers will be cancelled out by the incorporation of balancing modifiers either autosomal or linked to the opposite sex chromosome. Human geneticists have generally maintained that the tendency toward an excess of male births could have been brought about in response to differential mortality. Nevertheless, as will be argued later, this factor at best provides only a partial explanation of the observed ratio.

In all existing theoretical studies on human sex ratio, workers have tacitly assumed discrete time models. Schull (1972), however, has indicated that such an approach may lead to an erroneous approximation. Furthermore, for the study of the evolutionary process one must also consider the sex ratio at mating time, $\left(M_{m}\right)$. Because of the almost universal age difference that exists between the respective males and females at marriage, human mating groups generally consist of older bridegrooms and younger brides. Hence a balanced sex ratio in the mating group does not necessarily show himself in the demographic cohorts. Moreover, in a growing population an unbalanced sex ratio in the demographic cohort is a necessary condition for a balanced sex ratio in the mating group. I shall try to avoid the pitfalls inherent in the conventional representation of tertiary sex ratios in terms of separate age cohorts, and will take into account the important but neglected parameter $M_{m}$ : the sex ratio that is obtained in the actual mating group as a whole, including, as it does, different parts of several age cohorts.

I shall explore the effects of several demographic factors on $M_{m}$ :

(i) $M_{b}$, sex ratio at birth.

(ii) $R_{b}$, intrinsic growth rate of human populations as scored at birth.

(iii) $d$, age difference between males and females at marriage.

(iv) $L$, mortality.

(v) $F_{p}$, polygamy and polyandry.

In the analysis below, $d$ assumes a central importance. Furthermore, an attempt will be made to show that in humans, $d$ operates as a buffer to bring about a balanced $M_{m}$ in spite of the centrifugal pressures on this ratio that arise from changes in the values of the other five parameters considered. As a buffer, the system is asymmetrical, neutralising an excess of males more efficiently than an excess of females.

\section{Sex ratio at birth, $M_{b}$}

The demographic ratio, often regarded as the secondary sex ratio, may be expressed as the percentage of males among live births or, as in this paper, the number of males per 100 females, designated $M_{b}$. 
The available demographic records show that the present modal $M_{b}$ hovers around 106, a value obtained in many European countries, in the American Caucasian population (Lawrence, 1941), and among the Jews in Israel (Israel statistics, 1973). Among Cuban blacks and the Asians in South Africa, $M_{b}$ falls as low as 101 (Heape, 1908; Visaria, 1967), whereas in Greece, Korea, Aden and Gambia, its value is as high as 113-117 (Russel, 1936; Kang and Cho, 1962; Visaria, 1967). Visaria mentioned four small territories of predominantly black populations, with $M_{b} \leqq 100$. There is a considerable amount of evidence that the black race has a significantly lower $M_{b}$ than other races.

Edwards (1962) and Cavalli-Sforza and Bodmer (1971), concluded that shifts in sex ratio at birth can be achieved only after a long period of selection. These authors calculated that at least 2000 years are necessary to change the secondary sex ratio from $108 \cdot 3$ to $103 \cdot 0$. Hence, if $d$, the age difference at marriage, and $R_{b}$, population growth rate, and any other parameter to be described have had any influence on the evolution of the genetic control over the human secondary sex ratio, their action must have been effective for at least two millennia.

The earliest data on secondary sex ratio known to the author is that of London births between 1628 and 1710 (Arbuthnott, 1710), giving an average sex ratio of 106. A high sex ratio is, therefore, not a consequence of the demographic pressures of the last 350 years, under which the world population has been growing very rapidly. In the absence of accurate census data for the period before A.D. 1600, we must rely on cultural and historical evidence.

\section{Growth RAte AT BIRTH, $R_{b}$}

The symbol $R_{b}$ represents the increase or decrease in the number of births in a given human population, comparing subsequent age cohorts with their predecessors. When the newborns in year $i$ number $\mathcal{N}_{i}$, and in the subsequent year $\mathcal{N}_{(i+1)}$, then

$$
R_{b}=\frac{N_{(i+1)}}{N_{i}} .
$$

One should note that, in our usage, $R_{b}$ is independent of postnatal mortality, but whenever mortality values follow stable patterns, $R_{b}$ describes the general growth of the population involved. Only under such conditions is $R_{b}$ equivalent to $e^{m}, m$ being Fisher's malthusian parameter of population increase (Fisher, 1958).

Exact demographic data bearing on population increase are available only for the last few centuries. Demographers conventually estimate that world population doubled between A.D. 1 and A.D. 1650 (Dorn, 1962), i.e. an average increase of less than 0.05 per cent per year. However, human population growth in historical times has been a discontinuous process. Increases were interrupted by the common disasters of famine, plague and war. The usual pattern included a phase of gradual increase, terminated by an abrupt decimation and followed once more by a slow recovery. Thus, the actual $R_{b}$ during the prolonged recovery periods is considerably larger than the value derived from general data on population size. Moreover, a part of the decimation is equally distributed among all age cohorts, and has no impact on population age structure. Thus the total influence of $R_{b}$ must be 
significantly larger than the influence of the average $R_{b}$ calculated from the overall estimate of historical population growth. A conservative estimate of the effective $R_{b}$ in human populations would place it higher than 1.005, and very probably in the range of $1 \cdot 02-1 \cdot 04$. The significance of such values of $R_{b}$ for our model will be discussed below.

In addition, our ancestral population must have had even a larger population growth rate than the world average. There must have been some variation in the total growth of different human populations. Successful societies either suffered less from disasters or had higher $R_{b}$ 's, and are therefore the sources of present human populations.

\section{Age difference between mates, $d$}

The difference in age between mates is a parameter which has generally been neglected in the study of human sex ratios. The pattern of older husbands and younger wives is almost universal in human societies. In the first place, males reach sexual maturity at least 1-2 years later than females born at the same time. Second, the marriage of older bridegrooms to younger brides is traditional in most human societies. These are wellestablished historical patterns and are reflected in the marriage laws of the Roman, Ottoman and Jewish codes, in ancient literature and in relatively primitive contemporary cultures (Goode, 1963). Traditionally, the prospective bridegroom had first to establish himself economically, and only subsequently could he marry. In contrast, girls were married at as young an age as possible. Goode (1963) also disproves the commonly held notion that in ancient societies all marriages commenced when both the participants were very young. Thus even the ancients are seen to have had appreciable age differences between mates.

But, while it is clear that age differences at marriage are universal or nearly so, it is difficult to choose the suitable type of average age difference to represent the whole range of differences. The age difference in marriage of the younger women has more weight in evolutionary consideration than the difference in marriages of older women. For the present study we neglect such refinements, and use the simple " average age difference in first marriage "; including those that are the first only for one of the mates.

This average is designated by $d$, the average variable: bridegrooms' age minus the age of their brides.

\section{Mating Sex Ratio, $M_{m}$}

The concepts " mating group" and "mating sex ratio, $M_{m}$ " are defined in the introduction. It should be noted that $M_{m}$ is the ratio between the sexes in the mating group, including individuals of several age cohorts, and not necessarily only whole age cohorts. The mating group will comprise females of age $j$ and males of age $j+d$. Thus, only when we have some idea about the structure of the mating groups can the value $M_{m}$ be calculated. However, the exact $M_{m}$ is difficult to evaluate from demographic data, because of the overlap between mating groups caused by the variation in $d$. For qualitative treatment, it is enough to realise that in a strict monogamous society, if there is an excess of females in the mating group, $M_{m}<100$, while an excess of males would give $M_{m}>100$. In a polygamous society the 
balanced value of $M_{m}$ has to be calculated in a way presented in Section 8, below.

\section{Mortality, $L$, AND ITS EFFEGT}

Mortality may be regarded in connection with the ages of the individuals of both sexes comprising the mating group. Let $L$ represent the cumulative mortality fraction, then $L_{f j}$ will represent the total female mortality till marriage age $j$, and $L_{m(j+d)}$ the male mortality.

The influence of mortality on the mating sex-ratio $M_{m}$, is expressed by the ratio

$$
\frac{1-L_{m(j+d)}}{1-L_{f j}}
$$

Thus, even if both sexes have exactly equal mortality at all ages, the age difference at marriage $(d)$ will bring about a ratio lower than 1 . On the other hand, evidence will be presented that at least in some non-industrial societies the mortality ratio can attain values greater than one!

With the help of $L$, the tertiary demographic sex ratio can be computed from the demographic secondary sex ratio at birth, $\left(M_{b}\right)$, but for the computation of $M_{m}$, both $L$ and $d$ are necessary.

It should be noted that mortality after marriage has no direct impact on $M_{m}$, although it may affect the amount of vertical polygamy (and polyandry) and thus indirectly influence the value of $M_{m}$.

\section{The EFfects of POLYGaMy AND POLYANDRY}

Polygamy can affect the boundaries of the mating groups, tending to reduce the balanced $M_{m}$ (even without excesses of either sex) to values below 100. Polygamy will as a rule bring about an increase in $d$. Usually the subsequent wives are younger than the first ones. A second effect of polygamy might be a shortage of brides for other males, and that might bring about even older bridegrooms.

Although we include here only the polygamous marriages to a previously unmarried person, some types of "vertical polygamy" might have similar effect, i.e. a marriage between a widower or a divorced man to a previously unmarried woman. Obviously, "vertical polyandry" will have the opposite effect.

We consider the number of females entering polygamy (in their first marriage) per 100 oᄋ as $F_{p}$.

\section{INTERAGTIONS BETWEEN THE VARIOUS GOMPONENTS}

Let us first examine the interrelationships between $M_{m}, M_{b}, R_{b}$ and $d$, and then, for completeness, the effects of mortality and polygamy on these components. The interrelationships are as follows:

By definition of the parameters:

$$
\frac{M_{m}}{100}=\frac{N_{i} \cdot \frac{M_{b}}{M_{b}+100}}{N_{(i+d)} \frac{100}{M_{b}+100}}
$$


$\mathcal{N}_{i}$ is the number of newborns in year $i$. But $\mathcal{N}_{(i+d)}=\mathcal{N}_{i} \cdot R_{b}^{d}$, so

$$
\frac{M_{m}}{100}=\frac{M_{b} \cdot N_{i}}{100 \cdot N_{i} \cdot R_{b}^{d}} .
$$

If $\frac{M_{b}}{100}<R_{b}^{d}$, then $M_{m}$ is less than 100 (equation 2), and there is an excess of females in the mating group. The opposite will happen if $\frac{M_{b}}{100}>R_{b}^{d}$, an excess of males will exist in the mating group. For the balanced $M_{m}=100$ we get

or in logarithmic form

$$
M_{b}=100 R_{b}^{d}
$$

$$
d=\frac{\log M_{b}-2}{\log R_{b}} .
$$

Since $d$ in human societies is universally $>0$ and generally $R_{b}>1$, then $M_{m}=100$ will be realised only if $M_{b}>100$. In other words, in order to achieve 1 to 1 ratio at mating time in an increasing population, sex ratio at birth should be greater than 100 . This is, in fact, what we encounter in human populations, where, as already mentioned, values around $M_{b}=106$ are frequent in numerous societies.

If it is advantageous to achieve numerical equality at mating and if we assume that $M_{b}$ is genetically determined at the value of 106 , then to realise this equality $d$ and $R_{b}$ must vary as follows:

$$
\text { If } \begin{aligned}
R_{b} & =1 \cdot 005, \text { then } d=12 \text { years (exactly } 11.66 \text { ) or if } \\
R_{b} & =1 \cdot 01, \text { then } d=6 \text { years (exactly } 5 \cdot 86 \text { ) or if } \\
R_{b} & =1 \cdot 02, \text { then } d=3 \text { years (exactly } 2 \cdot 94 \text { ) or if } \\
R_{b} & =1.03, \text { then } d=2 \text { years (exactly } 1.97 \text { ). }
\end{aligned}
$$

All those values represent conditions that are most likely to happen in human populations.

Incorporating the factor of mortality, $L$, to the model, equation (2) becomes:

$$
\frac{M_{m}}{100}=\frac{M_{b} \cdot N_{i}}{100 \cdot N_{i} \cdot R_{b}^{d}} \cdot \frac{1-L_{m(j+d)}}{1-L_{f j}} .
$$

Assuming, as for equation (3), that $M_{m}=100$, we get

or in logarithmic form

$$
M_{b}=100 \cdot R_{b}^{d} \cdot \frac{1-L_{f j}}{1-L_{m(j+d)}}
$$

$$
d \log R_{b}=\log M_{b}-2+\log \left[\frac{1-L_{m(j+d)}}{1-L_{f j}}\right] .
$$

It should be noted that for any $\Delta R_{b}$ or $\Delta M_{b}$, that leads to an increase in $d$,

$$
\Delta d \text { (equation 4) }>\Delta d \text { (equation 7) }
$$

This inequality always holds when $j$ is held constant. Furthermore, for industrial societies there are extensive data indicating that $L_{f j}<L_{m j}$, in all 
ages before the reproductive period (Cavalli-Sforza and Bodmer, 1971) and even when $L_{f j}=L_{m j}, L_{f j}<L_{m(j+d)}$.

Whence $\log \left[\frac{1-L_{m(j+d)}}{1-L_{f j}}\right]<0$, and for a given $R_{b}$ and $M_{b}$,

$d$ (equation 4$)>d$ (equation 7).

In other words, mortality too demands $M_{b}>100$, in order to achieve $M_{m}=100$, and, therefore, a smaller age difference at marriage than that derived by $R_{b}$ alone, is sufficient to achieve a balanced $M_{m}$.

It is easily realised that when polygamy is practised, fewer males are needed to achieve a balanced ratio at marriage.

The balanced sex ratio at marriage under polygamy occurs when $\frac{M_{m}}{100-F_{p}}=1$, or $M_{m}=100-F_{p}$. Therefore, equation (7) becomes:

$$
d \log R_{b}=\log M_{b}-\log \left(100-F_{p}\right)+\log \left[\frac{1-L_{m(j+d)}}{1-L_{f j}}\right] .
$$

\section{Asymmetrical RANGe of the BUfFering efFect}

The fullest equation presented is equation (8), in which five factors interact to achieve a balanced sex ratio at mating, $M_{m}=100-F_{p}$. Two of these factors, $M_{b}$ and $R_{b}$, are fixed at birth. Although they may fluctuate from year to year, they cannot change for a given cohort. $L$ is also essentially fixed before the reproductive period has started. The values of $M_{b}, \mathrm{R}_{b}$, and $L$ are subject to evolutionary change under the impact of selection. In the reproductive stage $d$ and $F_{p}$ interact so as to buffer deviations, and to achieve, if possible, the balanced $M_{m}$. The exact values of $d$ and $F_{p}$ will reflect both the composition and the mores of the population.

In every society there is an age at which mate hunting commences. I will call the average age difference between the two sexes at this time the basic $d$. The process of looking for a mate will continue until all mating demands are satisfied or hope relinquished. In societies without polygamy the tendency is towards $M_{m}=100$. Where polygamy does exist, it will result in $M_{m}<100$.

Whether the balanced $M_{m}$ is achieved in reality depends on $R_{b}>100$ and on the ratio at the beginning of the mating period.

Assume a population with a culturally determined basic $d$. There are three possibilities at the start of the courting period:

(i) All mating demands are satisfied immediately: The basic $d$ will then be the realised $d$ and the group achieves its balanced $M_{m}$.

(ii) There is an initial excess of males: The unmated bachelors will then tend to wait for the females of the coming cohorts. In most cultures the male is evaluated in terms of his economic ability, either for the benefit of the new family or by the size of the bride price he can afford to pay. Usually his wealth and social status increases with time, at least for the first 15-20 years of adulthood. He is able to compete successfully against younger males and they, in turn, may have to wait until the older males are effectively out of the picture. This demographic pattern promotes an increase in $d$ and additional 
females are introduced into the mating group until the $M_{m}=100$ is realised.

The male is biologically endowed with a long reproductive period, enabling him to delay mating and reproduction if necessary, whereas in most cultures nothing deters the marriage of a young woman even to an old man. On the other hand, the period when males are waiting takes its toll in additional mortality and thereby helps reduce the male excess, but this effect will be significant only if $d$ is relatively large. In consequence, it is rare that $d$ reaches its maximum, and an unbuffered male excess develops. This, however, will happen in declining populations.

(iii) There is an excess of females: In this instance the outcome depends on $d$ of previous mating groups. When $d$ is larger than the basic $d$, waiting males are present and $d$ will decrease until it equals basic $d$. The process will continue as long as there are unmated males in the population who dip into the new supply of younger females, maintaining $M_{m}$ at balance.

However, from the moment that $d$ must decline below the "basic $d$ " the buffering process starts to be ineffective. As unmarried women wait for growing males, they come into ineffectual competition with women from the younger cohorts who grew up together with those males. In contrast to the effect of age on males, age is an important element in the marriage of women. In most cultures young brides are preferred and, inevitably, some of their older competitors will remain spinsters. If the female excess continues for a period of years, the " basic $d$ " may decrease under the onslaught of female attempts to find bridegrooms. Nevertheless, $d$ will not decrease to a negative or a zero value. The result of the cultural habits of mate preferences, is that brides older than their respective grooms, may occur as exceptional cases, but not as a general relationship.

In summary, the buffering is almost always efficient in neutralising male excesses, but adequate buffering of an excess of females, conditioned on a reduction of $d$ below the basic $d$ (equation 8), will only partly be achieved, as long as $d$ is maintained as a positive value, and conforms with cultural limitations.

This buffering process is independent of the cause of the initial excess of either sex. An excess of males may be caused by a higher $M_{b}$, a decrease in $R_{b}$, a change in mortality, less polygamy or a culturally decreed decrease in basic $d$. An opposite change in any one of those factors will produce a female excess. The demographic changes may represent random fluctuations or shifts in parameters without affecting the buffering process, except that $R_{b}>1.00$ is a necessary condition for the buffering of a parametrical shift. Schull (1972) pointed out that primitive societies must suffer from considerable fluctuations of population size. The sex ratio, $M_{b}$, has a considerable element of chance in its realisation and will therefore fluctuate seriously at times of small population size. In addition, drift may cause fixation of alleles affecting sex ratio, mortality and intrinsic growth rate, and thereby bring about permanent genetic change of the parameters. The buffering system enables the human society to function harmoniously in spite of the instability described, and hence must be advantageous in human evolution.

The biological and cultural limitations on $d$ are defining a multidimensional buffered range for all the interacting parameters. Tables 1 and 2 present some of the values of $M_{b}, R_{b}$ and $d$ that interact to produce $M_{m}=100$. The following points are evident: (a) When $R_{b}=1 \cdot 00$ (no population 
growth) then $M_{b}$ must be 100 , and deterministic changes cannot be buffered. (b) If $M_{b}=100$, and if $R_{b}=1 \cdot 00$, then $d$ can assume any value without any change in $M_{m}$, but if $R_{b} \neq 1.00$ with $M_{b}=100$, then $d$ must be zero, otherwise $M_{m}=100$ is not achieved.

The values in the tables are for deterministic models. For example, in a society which allows $d$ to vary from 2 to 10 years, and which is growing at a rate of $R_{b}=1 \cdot 02, M_{b}$ may be as small as 104 , or as large as 122 , without any impact on sex ratio at mating, $M_{m}$, because inside those limits, $d$ will be able to react appropriately.

\section{TABLE 1}

Values of sex ratio at birth $\left(\mathrm{M}_{\mathrm{b}}\right)$ that will produce a balanced sex ratio at mating, $\left(\mathrm{M}_{\mathrm{m}}=100\right)$, as a function of intrinsic growth rate at birth $\left(\mathrm{R}_{\mathrm{b}}\right)$ and age difference at marriage $(\mathrm{d})$

\begin{tabular}{ccccccccc}
\multicolumn{1}{c}{$d$} & 0 & 1 & 2 & 3 & 5 & 8 & 10 & 15 \\
$R_{b}$ & & & & & & & & \\
$1 \cdot 000$ & $100 \cdot 00$ & $100 \cdot 00$ & $100 \cdot 00$ & $100 \cdot 00$ & $100 \cdot 00$ & $100 \cdot 00$ & $100 \cdot 00$ & $100 \cdot 00$ \\
$1 \cdot 005$ & $100 \cdot 00$ & $100 \cdot 50$ & $101 \cdot 00$ & $101 \cdot 51$ & $102 \cdot 53$ & $104 \cdot 10$ & $105 \cdot 11$ & $107 \cdot 77$ \\
1.010 & $100 \cdot 00$ & $101 \cdot 00$ & $102 \cdot 01$ & $103 \cdot 03$ & $105 \cdot 10$ & $108 \cdot 29$ & $110 \cdot 46$ & $116 \cdot 10$ \\
$1 \cdot 020$ & $100 \cdot 00$ & $102 \cdot 00$ & $104 \cdot 04$ & $106 \cdot 12$ & $110 \cdot 41$ & $117 \cdot 17$ & 121.91 & $134 \cdot 57$ \\
$1 \cdot 030$ & $100 \cdot 00$ & 103.00 & $106 \cdot 09$ & $109 \cdot 27$ & $115 \cdot 93$ & $126 \cdot 68$ & $134 \cdot 39$ & $155 \cdot 80$
\end{tabular}

TABLE 2

Age differences at marriage (d) that will produce a balanced sex ratio at mating $\left(\mathrm{M}_{\mathrm{m}}=100\right)$, as a function of sex ratio at birth $\left(\mathbf{M}_{\mathfrak{b}}\right)$ and intrinsic growth rate at birth $\left(\mathbf{R}_{\mathrm{b}}\right)$

\begin{tabular}{|c|c|c|c|c|c|c|c|c|}
\hline$M_{b}$ & 100 & 101 & 102 & 103 & 106 & 110 & 113 & 120 \\
\hline $\begin{array}{l}R_{b} \\
1.000\end{array}$ & & no $d$ & no $d$ & no $d$ & no $d$ & nod & no $d$ & no $d$ \\
\hline 1.005 & 0 & $2 \cdot 00$ & 3.97 & $5 \cdot 93$ & 11.68 & $19 \cdot 11$ & $24 \cdot 50$ & $36 \cdot 56$ \\
\hline 1.010 & 0 & 1.00 & 1.99 & $2 \cdot 97$ & $5 \cdot 86$ & $9 \cdot 58$ & $12 \cdot 28$ & $18 \cdot 32$ \\
\hline 1.020 & 0 & 0.50 & 1.00 & $1 \cdot 49$ & 2.94 & $4 \cdot 81$ & $6 \cdot 17$ & $9 \cdot 21$ \\
\hline 1.030 & 0 & 0.34 & 0.67 & 1.00 & 1.97 & $3 \cdot 22$ & $4 \cdot 13$ & $6 \cdot 17$ \\
\hline
\end{tabular}

When dealing with small populations, we must introduce stochastic processes. Every parameter will have a range of fluctuations in time, which must entirely be included inside the range of the buffered system, or else the population will come to include an excess of one sex or the other beyond the amount that can be neutralised as a change in $d$. This circumstance reduces the size of the buffered region for all the parameters.

Concerning the effects of mortality on balancing sex ratio, it is doubtful whether this factor has unidirectional effect in man's history. However, if $L_{f j}<L_{m(j+d)}$, then the $M_{b}$ values in table 1 must be larger, and in table 2 the $d$ values will be smaller.

Mortality operates on the waiting males in proportion to their age. Thus its impact is proportional to the size of $d$. The limits of $d$ are culturally and biologically determined and these consequently also establish the limits of the buffered range. Therefore mortality increases the buffered range, in addition to the shift of both limits of the range towards higher values of $M_{b}$ and $R_{b}$.

The dual effects of polygamy on the buffered range include a decrease in the value of $M_{m}$ at balance and an increase in $d$ in consequence of the conventionally younger age of second and subsequent wives. 


\section{The evolutionary significance of EXgess male births}

There are two main types of explanation for the evolution of sex ratio: (i) "Balanced models" which assume a process of selection by individual fitness and the achievement of an equilibrium between two antagonistic pressures: one pressing for a high sex ratio, the other for a low one. "Optimum models" which stress kin or group selection.

Both types of selection may have operated in man. The critical question is the relative weight that should be accorded to each. Since the expectations from the two types of models differ, an analysis of the components that may have influenced to the existing sex ratio can provide clues as to their relative importance.

\section{(i) Balanced or fitness models}

The simplest and basic "balanced model" is the treatment which assumed the existence of alleles for deviations of sex ratio (Crow and Kimura, 1970). Eshel (1973) has developed this model to include any kind of " modifiers of sex ratio" and showed that the ratio tends to converge to an equilibrium at $1: 1$. Any deviation from this ratio results in an expectation of more grandchildren for the genotype that is correcting the sex ratio towards the 1:1 ratio. Since this analysis predicts the sex ratio of $1: 1$ to be attained in the zygotes, mortality and polygamy do not change the expectations.

However, $d$ does influence the lapse of time until the expectations of grandchildren are realised. Families with relative lower sex ratio beget grandchildren at an earlier age, i.e. a shorter generation time, implying a higher fitness of such genotypes in an increasing population. To balance that advantage in favour of females, an equilibrium with excess of females, a low sex ratio will evolve. Only then will the female advantage of shorter generations be balanced by the male advantage deriving from rarity. In other words, on the basis of individual fitness models one actually expects a small excess of females and not equality. The observed excess of males in human populations is, therefore, even more significant.

\section{(ii) Sex-linked modifiers}

Hamilton (1967) and Eshel (1973) showed that sex-linked modifiers in the long run will also tend to converge on the $1: 1$ ratio. Modifiers that increase the frequency of the same sex chromosome to which they are linked will spread in the population. But the introduction of such modifiers will probably be followed by the subsequent appearance and establishment of an antagonistic modifier, linked to the opposite sex chromosome or carried on an autosomal chromosome. The buffering effect thus helps to guard against an "aggressive" Y chromosome. A Y chromosome which confers a moderately higher sex ratio will cause only an increase in $d$. However, if the effect of the $\mathrm{Y}$ on the sex ratio is more drastic and the sex ratio is too high to be buffered by $d$, $d$ will at least reach its maximum. In that event, the $\mathrm{X}$ chromosomes in the females will have a quicker turnover and more generations for the development and selection of a balancing factor, either an " aggressive" $\mathrm{X}$ or a female favouring autosome. 


\section{(iii) Optimum models and differential mortality}

The previously discussed models consider the expectations of sex ratio in utero. In contrast, Fisher's expenditure model (Fisher, 1958) and all optimum models predict equilibrium at tertiary sex ratio, and all predict a $1: 1$ ratio or even an excess of females. If this happens and males suffer a relatively high mortality rate, sex ratio at birth should indeed show an excess of males. This argument has already been brought up by Arbuthnott (1710), who considered the male excess a result of " divine providence", because "The male has to look for his food with danger".

Differential mortality has been seriously reviewed by Cavalli-Sforza and Bodmer (1971). They calculated that differential mortality of 1805 transforms a $M_{b}=103$ to a tertiary $M_{b}=100 \cdot 8$. But since this reduction is not enough to account for the even higher existing $M_{b}$ 's, they extrapolate: Mortality is much higher in non-industrial than in industrial societies. If differential mortality is similar in primitive societies, then it has a much stronger impact on sex ratio. The extrapolation is not generally applicable. An opposite trend was demonstrated in data from India (Gopalan and Nadamuni, 1972), where most of the population live " non-industrial" lives. Girls and younger women there have a higher mortality rate than boys, and the sex ratio increases constantly from the age of one year to late in the reproductive period. This shows that cultural and behavioural habits play an essential part in differential mortality. During the last several millennia, human society was predominantly patriarchal and may have followed the Indian pattern of differential mortality.

In its present form differential mortality does not adequately explain the whole phenomenon of high sex ratio. The extrapolation towards primitive societies is dubious and certainly cannot be true for all societies. It was put forward for lack of any other attractive explanation. Equation 8 includes both differential mortality and $d$. If mortality had been enough to neutralise the excess male births, then $d$ would have caused a constant excess of females, and there is no reason why that age difference was established in the past. Thus, $d$ and mortality must be two complementary parts of the explanation, as their influence is in the same direction, or even, as in the case of India, $d$ must adjust to outbalance an antagonistic differential mortality, an excess of girl mortality.

\section{(iv) Indication for group selection}

"Optimum selection models" imply some kind of group and/or kin selection. Only these models consider sex ratio at mating as the element for selection. In fitness models the goal of selection is sex ratio in utero, and Fisher's expenditure model considers the ratio at childhood. The interactions described here buffer the sex ratio at mating only.

In a Darwinian fitness model of a growing population, a selective disadvantage accrues from the additional time a parent must wait for grandchildren from sons as compared to daughters. This disadvantage of $d$ should have acted in favour of a low ratio, or for $d=0$. The fact that we encounter an opposite situation in man can best be explained in terms of selective advantage for the whole group, i.e. by "optimum models". Indeed, this is not surprising, as the organisation of mankind into competing clans, families, tribes or villages provides the necessary conditions for the operation of group selection. 


\section{Selection Within the BUfFering RANGe}

Within the buffering range the mechanism of selection is different from outside it. Outside the range, the excesses of one sex or the other at mating generate the selection pressures. Within the buffering range there are no excesses. Instead, the longer male generation time will lead to selective pressures. The tendency will be toward a shortening of generation time, thus causing a reduction in $d$. This reduction may be a very slow process, since $M_{b}$ reacts slowly, and both $R_{b}$ and $L$ may depend upon environmental factors resistant to change. $d$ may therefore remain relatively large for numerous generations, although selection will tend to reduce its value, as, for example, by lowering $M_{b}$. The reduction of $d$ will be stopped near the basic $d$. Some eligible brides will be left without mates, leading to a decrease in $R_{b}$, since some fertile women will be left outside the reproductive process. In other words, an excess of females slows a population increase in the next generation. A decrease in $R_{b}$ will produce an excess of males, and $d$ will be pushed back into the buffering zone. Such fluctuations of $d$ may continue for a long time and prevent further decrease of $d$.

The situation in Israel during the last few years can serve as an example of exclusion of women from marriage and reproduction instead of a decline of $d$.

The "baby booms" after World War II and Israel's War of Independence produced an increase in the 5-year cohorts in the Jewish population in Israel. The cohorts born before 1943, 145,000 people, and those born post1948 include 265,000 , equivalent to $R_{b}=1.062$ for 10 years of continuous population growth, or to $R_{b}=1 \cdot 128$ if the growth period was of 5 years only. The demographic sex ratio, in the relevant age cohorts, is 103.6 and 104.2 (Israel's statistics, 1973). Therefore, $d$ should fall between five and ten months in order to achieve a balance between the two sexes. In fact, $d$ is approximately 2.9 years and, consequently, an unfortunate lack of bachelors is publicly felt in spite of the actual excess of males. It is generally known that spinsterhood is common, but statistical documentation will become available a few years hence. Thus the steep increase in population size will be transformed into a more gradual increase in the next generation, because the unmarried women contribute to a decrease in $R_{b}$.

To summarise: $R_{b}$ adjusts earlier than $M_{b}$. In contrast, millennia are required for the introduction of a significant change in $M_{b}$, and by that time the pressures in favour of a decrease in $d$ are usually relieved.

\section{2. $d$ IN DEGLINING POPULATIONS}

In this discussion we consider a continuous decrease, i.e. a negative $R_{b}$ : a decline in the number of newborns every year. Because the inherited $M_{b}$ is high, this can be buffered only by a negative $d$, i.e. by marriage between younger males and older females, a custom that is not generally practised in most cultures.

Initiation of a population decline will bring about an excess of males and, therefore, an increase in $d$. Since the subsequent cohorts will have even smaller numbers of females, the male excess will tend to accumulate until $d$ achieves its maximum attainable value, and an unbuffered male excess develops. There is no way to reverse this process to bring about a negative $d$. 
The only solution is a reversal of $R_{b}$, or a sharp decrease in $M_{b}$, but neither of these normally takes place. It is important to remember that the buffering mechanism evolved in increasing human populations, and not in any decreasing population. Most of the latter very likely have vanished. A possible example is found in the Samaritans, who have been reduced to only two hundred persons. Their religion prohibits marrying women from outside their caste. They provide indeed an example of maximum $d$. Adult Samaritan males often await the outcome of every birth in their community. If the child is a girl, the event may solve an individual personal problem (information from personal visit). That situation indeed imposes considerable social hardship on the Samaritan community.

\section{OTHER SPECIES}

The question now arises whether an age-difference buffering system will be found in species other than man. The problem of sex ratio distortions will exist whenever a population is organised in small groups, as in such cases serious fluctuations in sex ratio can arise through chance and genetic drift. An age difference buffering system will be feasible whenever the population increases steadily in numbers, or at least has relatively continuous increases between disasters. However, under such conditions other solutions are also possible. Among them we can mention: dominant male herds, or efficient migration systems. Species practising the dominant-male solution are well known. Usually, however, we lack information as to the exact ages of the mates in free living animals. Therefore, if an age difference buffering system does exist, it is likely to escape our attention.

The sole, or at least the most common solution in humans is the age difference buffering system, rather than the dominant male-herd organisation typical of other primates. Is that strategy a cause, a consequence or merely a by-product of the rapidity of human evolution? This is an interesting problem, but beyond the scope of this paper.

Acknowledgments.--I am grateful to Professors B. Dorfman, R. Falk, M. Soller and D. Zohary for their valuable comments and criticisms of the manuscript, and to Dr Tirza Cohen for helpful references.

\section{RefERENGES}

ARBUTHNotT, I. 1710. An argument for Divine Providence, taken from the constant regularity observed in the births of both sexes. Philos. Trans. Roy. Soc. London, 27, 186-190.

CAVAlli-SFORZA, L. L., AND BODMER, w. F. 1971. The Genetics of Human Populations, pp. 666-

670. W. H. Freeman and Co., San Francisco.

crow, I. F., AND knMURA, M. 1970. An Introduction to Population Genetics Theory, pp. 288-293.

Harper \& Row, New York.

DORN, H. F. 1962. World population growth, an international dilemma. Science, 135, 283-290.

EDWARDS, A. W. F. 1962. Genetics and the human sex ratio. Adv. in Genet., 11, 239-272. ESHEL, I. 1973. Selection of sex-ratio and the evolution of sex-determination. Preprint. FISHER, R. 1958. The Genetical Theory of Natural Selection (Second Edition), pp. 158-162.

Dover Publications, Inc., New York.

GOODE, w. 1. 1963. World Revolution and Family Patterns. The Free Press, New York.

GOPALAN, C., AND NADAMUNI, NAIDU, A. 1972. Nutrition and fertility. The Lancet, 7786,

(Vol. II for 1972), 1077-1079.

haynemann, N. 1973. Chromosome studies in induced abortions. Clin. Genet., 4, 328-332.

$33 / 2-\mathrm{S}$ 
hamlton, w. D. 1967. Extraordinary sex ratios. Science, 156, 477-488.

HEAPE, $w$. 1908. The proportion of the sexes produced in white and coloured people in Cuba. Proc. Phil. Roy. Soc. London, B., 81, 32-37.

ISRAEL's CENTRAL BUREAU of STATISTICS. 1973. Statistical Abstracts of Israel. The Central Bureau of Statistics, Jerusalem.

KANG, Y. s., AND CHO, w. K. 1962. The sex ratio at birth and other attributes of the newborn from maternity hospitals in Korea. Human Biol., 34, 38-48.

Keller, C. A. 1969. Embryonal sex ratios in animals and man. Ph.D. Thesis, University of California, Berkeley.

LAWRENCE, P. s. 1941. The sex ratio, fertility and ancestral longevity. Quart. Rev. Biol., 16, 35-79.

MIKAMO, K. 1969. Prenatal sex ratio in man, observations contradictory to the prevailing concept. Obstet. and Gynec., 34, 710-716.

RUSSEL, w. T. 1936. Statistical study of the sex ratio at birth. Four. Hyg., 36, 381-401.

SASAKI, M., IKEUCHI, T., OBARA, Y., haYATA, I., MORI, M., AND KOHNO, S-I. 1971. Chromosome studies in early embryogenesis. Amer. F. Obstet. Gynec., 111, 8-12.

SCHULL, W. J. 1972. Primitive populations-some contributions to the understanding of human population genetics. Proc. 4th Inter. Cong. Hum. Genet., Paris 1971, 112-130.

STERN, c. 1973. Principles of Human Genetics (3rd ed.), pp. 529-551. W. H. Freeman and Co., San Francisco.

STEVENSON, A. C., AND BOBROW, M. 1967. Determinants of sex proportions in man, with consideration of the evidence concerning a contribution from X-linked mutations to intrauterine death. F. Med. Genet., 4, 190-221.

VISARIA, P. M. 1967. Sex ratio at birth in territories with a relatively complete registration. Eugen. Quart., 14, 132-142. 